This item was submitted to Loughborough's Research Repository by the author.

Items in Figshare are protected by copyright, with all rights reserved, unless otherwise indicated.

\title{
On stability of relaxive systems described by polynomials with time-variant
} coefficients

PLEASE CITE THE PUBLISHED VERSION

PUBLISHER

(c) IEEE

VERSION

VoR (Version of Record)

\section{LICENCE}

CC BY-NC-ND 4.0

\section{REPOSITORY RECORD}

Mandic, Danilo P., and Jonathon Chambers. 2019. "On Stability of Relaxive Systems Described by Polynomials with Time-variant Coefficients". figshare. https://hdl.handle.net/2134/5791. 
This item was submitted to Loughborough's Institutional Repository (https://dspace.lboro.ac.uk/) by the author and is made available under the following Creative Commons Licence conditions.

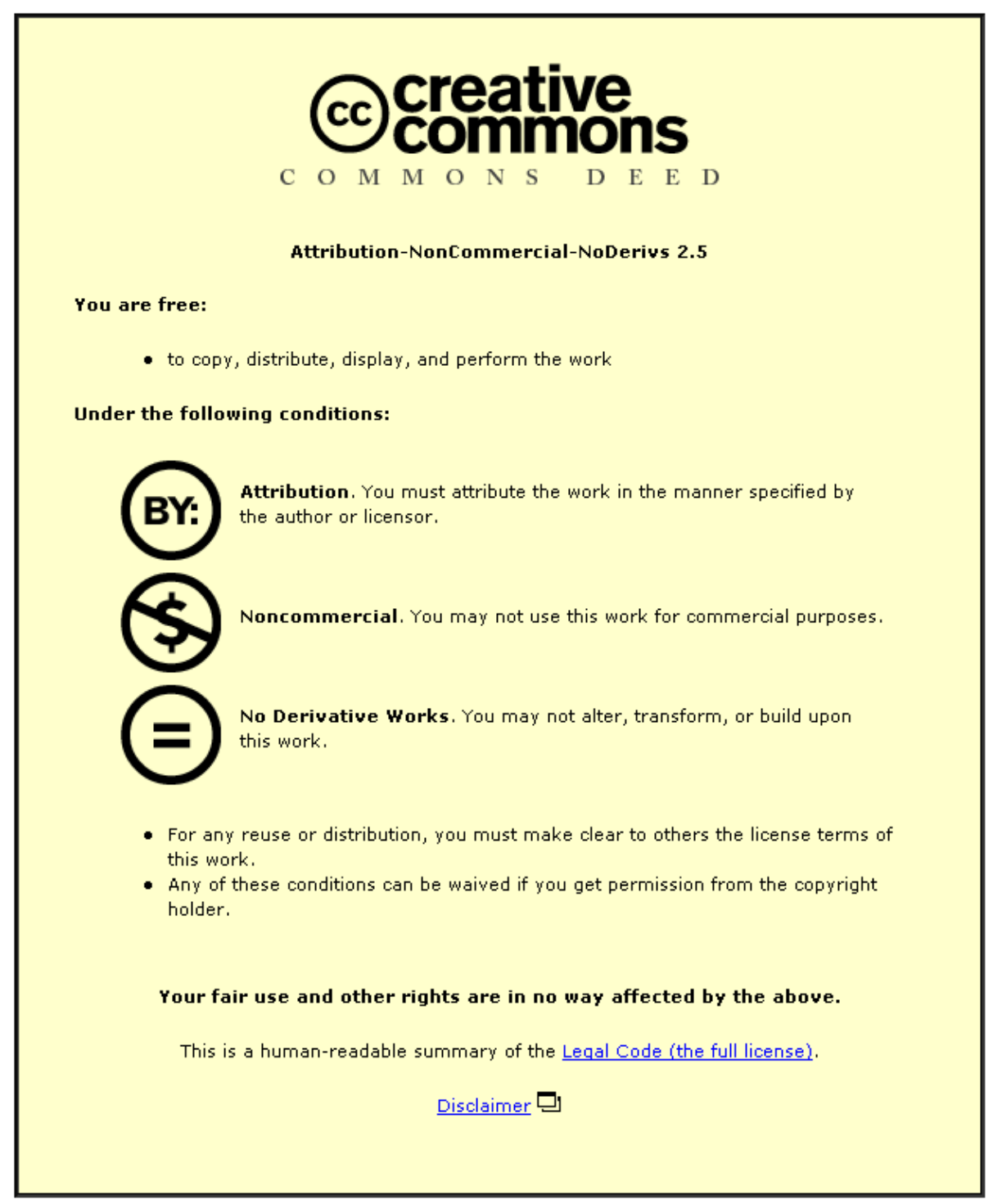

For the full text of this licence, please go to: http://creativecommons.org/licenses/by-nc-nd/2.5/ 
Proof: For 1), recall the assignment $\phi$ in the proof of Theorem 3.1. For 2), recall that the labels used in the optimal labeling of $C_{m_{0}} \square \cdots \square C_{m_{k-1}}$ in the proof of Theorem 2.1 are consecutive. For 3), recall the statement and proof of Corollary 2.2 .

\section{ACKNOWLEDGMENT}

The author would like to thank Dr. R. Balakrishnan and Dr. P. Paulraja, whose hospitality at Annamalai University, India, during July 1995 , led to his introduction to the topic of $L(2,1)$-labeling.

\section{REFERENCES}

[1] W. K. Hale, "Frequency assignment: Theory and application," Proc. IEEE, vol. 68, pp. 1497-1514, 1980.

[2] F. S. Roberts, " $T$-colorings of graphs: Recent results and open problems," Discrete Math., vol. 93, pp. 229-245, 1991.

[3] J. R. Griggs and R. K. Yeh, "Labeling graphs with a condition at distance two," SIAM J. Disc. Math., vol. 5, pp. 586-595, 1992.

[4] J. P. Georges, D. W. Mauro, and M. A. Whittlesey, "Relating path coverings to vertex labelings with a condition at distance two," Discrete Math., vol. 135, pp. 103-111, 1994.

[5] M. A. Whittlesey, J. P. Georges, and D. W. Mauro, "On the $\lambda$-number of $Q_{n}$ and related graphs," SIAM J. Disc. Math., vol. 8, pp. 499-506, 1995.

[6] G. J. Chang and D. Kuo, "The L $(2,1)$-labeling on graphs," SIAM J. Disc. Math., vol. 9, pp. 309-316, 1996.

[7] D. D.-F. Liu and R. K. Yeh, "On distance-two labelings of graphs," Ars Combin., vol. 47, pp. 13-22, 1997.

[8] D. G. Corneil and Y. Perl, "Clustering and domination in perfect graphs," Discrete Appl. Math., vol. 9, pp. 27-39, 1984.

[9] S. Klavžar and N. Seifter, "Dominating Cartesian products of cycles," Discrete Appl. Math., vol. 59, pp. 129-136, 1995.

[10] B. Alspach, J.-C. Bermond, and D. Sotteau et al., "Decompositions into cycles I: Hamilton decompositions," in Cycles and Rays, G. Hahn et al., Eds. Norwell, MA: Kluwer, 1990, pp. 9-18.

[11] D. Sakai, "Labeling chordal graphs with a condition at distance two," SIAM J. Disc. Math., vol. 7, pp. 133-140, 1994.

[12] J. van den Heuvel, R. A. Leese, and M. A. Shepherd, "Graph labeling and radio channel assignment," J. Graph Theory, to be published.

\section{On Stability of Relaxive Systems Described by Polynomials with Time-Variant Coefficients}

\author{
Danilo P. Mandic and Jonathon A. Chambers
}

Abstract-The problem of global asymptotic stability (GAS) of a timevariant $m$-th order difference equation

$$
\begin{aligned}
\boldsymbol{y}(n)= & \boldsymbol{a}^{T}(n) \boldsymbol{y}(n-1)=\boldsymbol{a}_{1}(n) y(n-1)+\cdots \\
& +\boldsymbol{a}_{m}(n) y(n-m)
\end{aligned}
$$

for $\|\boldsymbol{a}(n)\|_{1}<1$ was addressed in [1], whereas the case $\|\boldsymbol{a}(n)\|_{1}=1$ has been left as an open question. Here, we impose the condition of convexity on the set $\mathcal{C}_{0}$ of the initial values $y(n)=[y(n-1), \cdots, y(n-$ $m)]^{T} \in \mathbb{R}^{m}$ and on the set $\mathcal{A} \in \mathbb{R}^{m}$ of all allowable values of $a(n)=$ $\left[a_{1}(n), \cdots, a_{m}(n)\right]^{T}$, and derive the results from [1] for $a_{i} \geq 0, i=$ $1, \cdots, n$, as a pure consequence of convexity of the sets $\mathcal{C}_{0}$ and $\mathcal{A}$. Based upon convexity and the fixed-point iteration (FPI) technique, further GAS results for both $\|\boldsymbol{a}(n)\|_{1}<1$, and $\|\boldsymbol{a}(n)\|_{1}=1$ are derived. The issues of convergence in norm, and geometric convergence are tackled.

Index Terms-Contraction mapping, convergence, fixed-point iteration, global asymptotic stability, linear systems, relaxation.

\section{INTRODUCTION}

The issue of global asymptotic stability (GAS) of

$$
\begin{aligned}
\boldsymbol{y}(n)= & \boldsymbol{a}^{T}(n) \boldsymbol{y}(n-1)=a_{1}(n) y(n-1)+\cdots \\
& +a_{m}(n) y(n-m)
\end{aligned}
$$

is important in the theory of linear systems [2]-[4]. Equation (1) represents an autonomous system, which under certain conditions converges. Actually, it is a relaxation equation, which stems from a general linear system

$$
\boldsymbol{Y}(n+1)=\boldsymbol{A}(n) \boldsymbol{Y}(n)+\boldsymbol{B}(n) \boldsymbol{u}(n)
$$

for the zero exogenous input vector $\boldsymbol{u}(n)=\mathbf{o}, \forall n$ [2], [4]. Equation (1) can be further written in the state-space form as

$$
\begin{aligned}
{\left[\begin{array}{c}
y(n+1) \\
y(n) \\
\vdots \\
y(n-m+1)
\end{array}\right]=} & {\left[\begin{array}{cccc}
a_{1}(n) & a_{2}(n) & \cdots & a_{m}(n) \\
1 & 0 & \cdots & 0 \\
\vdots & \vdots & \ddots & \vdots \\
0 & \cdots & 1 & 0
\end{array}\right] } \\
& \cdot\left[\begin{array}{c}
y(n) \\
y(n-1) \\
\vdots \\
y(n-m)
\end{array}\right]
\end{aligned}
$$

with $y(n+1)=\left[\begin{array}{llll}1 & 0 & \cdots & 0\end{array}\right] \boldsymbol{Y}(n+1)$. Matrix $\boldsymbol{A}$, where the index " $n$ " is dropped for convenience, is a Frobenius matrix, which is a special form of the companion matrix of the characteristic polynomial [5], [6]. Namely, let us denote the characteristic equation of a general matrix $M$ by $(-1)^{n}\left[\lambda^{n}-p_{n} \lambda^{n-1}-\cdots-p_{0}\right]=0$, then, the characteristic equation of $\boldsymbol{A}$ (3) is identical to the characteristic equation of $\boldsymbol{M}$, and the matrix $\boldsymbol{A}$ is called the companion matrix of the characteristic polynomial of $\boldsymbol{M}$. Since $\boldsymbol{M}$ and $\boldsymbol{A}$ have the same characteristic polynomial, it

Manuscript received July 15, 1998; revised February 1, 1999

D. P. Mandic is with the School of Information Systems, University of East Anglia, Norwich, U.K.

J. A. Chambers is with the Department of Electrical and Electronic Engineering, Imperial College of Science, Technology and Medicine, London, U.K. Publisher Item Identifier S 1057-7122(00)09414-9. 
is natural to ask whether they are, in general, similar. Similar matrices have the same trace, determinant, characteristic polynomial, and eigenvalues [6]. The answer to this question lies in the fundamental theorem of general matrices [5], [6], which states that every matrix $A$ can be reduced by a similarity transformation [5], [3] to the direct sum of a number of Frobenius matrices. That is why it is important to consider the stability results for the Frobenius matrix $\boldsymbol{A}$ (3), since a stability result of a general system $\boldsymbol{C x}=\boldsymbol{y}$ can be obtained through the stability result of (3)

In this work, we show that the sequence of the sets $\mathcal{C}_{i} \in \mathbb{R}^{m}, i=$ $1,2, \cdots$ of the values of $\boldsymbol{y}(n)$ must, in the case of GAS, exhibit contraction features, which preserves convexity of adjacent subsets, as well as the asymptotic stability. Namely, based upon the Cauchy-Schwarz inequality

$$
|y(n)|=\left|\boldsymbol{a}^{T}(n) \boldsymbol{y}(n-1)\right| \leq\|\boldsymbol{a}(n)\|_{2}\|\boldsymbol{y}(n-1)\|_{2}
$$

which exhibits contraction features under the assumption $\|\boldsymbol{a}(n)\|_{2}<$ 1 .

Moreover, we derive the conditions for the pseudoperiodic or aperiodic GAS based upon the fixed-point iteration (FPI) technique. The conditions of the convergence of the FPI are based upon the $\|\cdot\|_{1}$ and $\|\cdot\|_{\infty}$ norms of the coefficient vector $\boldsymbol{a}$.

\section{INTERVAL CONTRACTION AND CONTRACTION MAPPING}

We now cite the contraction mapping theorem (CMT) for the univariate case [7], [8]

Theorem 1: If

i) $x \in[a, b] \Rightarrow K(x) \in[a, b]$

ii) $\exists \gamma<1$ s.t. $|K(x)-K(y)| \leq \gamma|x-y|, \forall x, y \in[a, b]$

then the equation $x=K(x)$ has a unique solution $x^{*} \in[a, b]$ and the iteration

$$
x_{i+1}=K\left(x_{i}\right)
$$

converges to $x^{*}$ from any $x_{0} \in[a, b]$.

The contraction feature can be seen as the interval contraction [9], whose convergence can be derived as

$$
\left|x_{i}-x^{*}\right|=\left|K\left(x_{i-1}\right)-K\left(x^{*}\right)\right| \leq \gamma\left|x_{i-1}-x^{*}\right|
$$

thus $\left|x_{i}-x^{*}\right| \leq \gamma^{i}\left|x_{0}-x^{*}\right|$ since $\left\{\gamma^{i}\right\}_{i} \rightarrow 0 \Rightarrow\left\{x_{i}\right\}_{i} \rightarrow x^{*}$ as $i \rightarrow \infty$. The usual way of checking ii) in Theorem 1 is to show that $\left|K^{\prime}(x)\right| \leq \gamma<1$, where $(\cdot)^{\prime}$ denotes the first order differentiation.

\section{COMMENTS ON THE GAS}

In [1], Lemma 1 stated that the time variant difference equation $\{(1)\}$ is asymptotically stable, if and only if $\Sigma_{v=1}^{m} a_{v}^{+}<1$, where $\left|a_{v}(n)\right| \leq$ $a_{v}^{+}, v=1, \cdots, m, \forall n$.

This was illustrated for the second order case. The illustration provided was very well known from autoregressive (AR) model theory [10], [11]. However, nothing was said about the set $\mathcal{C}_{n-1} \subset \mathbb{R}^{m}$ to which the vector $\boldsymbol{y}(n-1)=[y(n-1), \cdots, y(n-m)]^{T}$ should belong, nor to which set the resulting value $y(n)$ belongs, and whether the vector $\boldsymbol{a}(n)$ is allowed to change continuously with $n$ or not, and what the bounds on the set $\mathcal{A}$ would be for all $\{\boldsymbol{a}(n)\}$ in that case.

We should re-state this Lemma, so that it comprises the bounds on the set of initial values $\mathcal{C}_{0}$ and includes a continuous change of the parameter vector $\boldsymbol{a}(n)$ within its domain.

Lemma 1: Let the initial values $y(-1), \cdots, y(-m)$ of (1) belong to a convex set $\mathcal{C}_{0} \subset \mathbb{R}^{m}$, and $a_{v} \geq 0,\left|a_{v}(n)\right| \leq a_{v}^{+}$, for $v=1, \cdots, m$ and $\forall n$. Then the difference equation (1) is asymptotically stable if and only if $\sum_{v=1}^{m} a_{v}^{+}<1$, and the resulting value $y(n)$ belongs to a subset $\mathcal{C} \subseteq \mathcal{C}_{0}$ of the set $\mathcal{C}_{0}$.

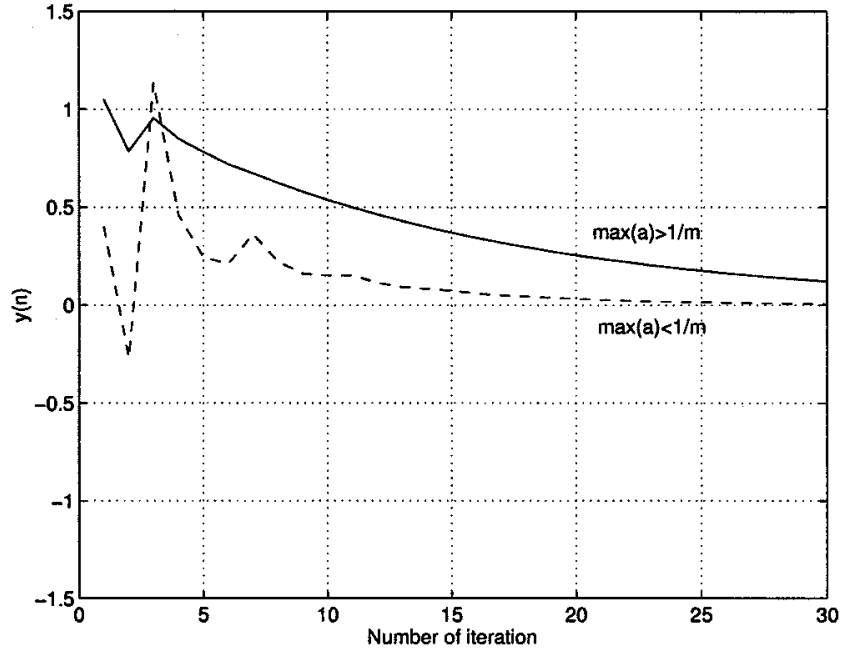

Fig. 1. Convergence of relaxation for $m=4$, the cases $\|\boldsymbol{a}\|_{\infty}>1 / 4$, and $\|\boldsymbol{a}\|_{\infty}<1 / 4$.

Proof: Affine function (1) is either convex or concave. If the set $\mathcal{C}_{0}$ is convex, and with the constraints on $\mathcal{A}$ as above, then the resulting value $y(n)$ belongs to the convex subset of $\mathcal{C}_{0}$. Now, the difference equation (1) is asymptotically stable, as a mere consequence of convexity and contraction mapping.

Furthermore the parameter set $\boldsymbol{a}(n)$ forms a closed halfspace after each iteration. That means that on the run, the set $\{\boldsymbol{a}(n)\}$ forms a polyhedron, which is convex.

\section{PSEUdoPERIOdIC AND APERIODIC CONVERGENCE}

The GAS results as introduced in [1] allow pseudoperiodic behavior, since there is no further condition on $\boldsymbol{a}(n)$, except for GAS. Let us present the result for the strict aperiodic uniform convergence of (1).

Theorem 2: The equation

$$
y(n)=a_{1}(n) y(n-1)+\cdots+a_{m}(n) y(n-m)
$$

exhibits uniform GAS in the aperiodic sense if

i) $a_{i}(n) \geq 0, i=1, \cdots, m, \forall n$

ii) $\sum_{i=1}^{m} \bar{a}_{i}(n)<1 \Leftrightarrow\|a\|_{1}<1$

iii) $\max _{a_{i}(n), i=1, \cdots m} a_{i}(n)>(1 / m) \Leftrightarrow\|\boldsymbol{a}\|_{\infty}>(1 / m)$

Proof: Points i) and ii) have already been considered.

iii) In order to preserve contraction of the sets $\mathcal{C}_{0}, \mathcal{C}_{1}, \cdots$, we have

$$
\begin{aligned}
|y(n)| & =\left|a_{1}(n) y(n-1)+\cdots+a_{m}(n) y(n-m)\right| \\
& \leq a_{1}|y(n-1)|+\cdots+a_{m}|y(n-m)| \\
& <\left(a_{1}(n)+\cdots a_{m}(n)\right)|y(n-1)| \\
& <m \cdot \max _{a_{i}(n), i=1, \cdots m} a_{i}(n)|y(n-1)|
\end{aligned}
$$

From (8) we have $\max _{a_{i}(n), i=1, \cdots m} a_{i}(n)>(1 / m)$.

Example 1: Check Theorem 2 for $m=4$ and

i) $[$ i) $] \boldsymbol{a}=\left[\begin{array}{llll}0.7 & 0.1 & 0.05 & 0.05\end{array}\right]$ where $\|a\|_{1}=0.9<1$ and $\max \boldsymbol{a}=\|\boldsymbol{a}\|_{\infty}=0.7>1 / 4$

ii) $\boldsymbol{a}=\left[\begin{array}{llll}0.2 & 0.1 & 0.15 & 0.2\end{array}\right]$ where $\|a\|_{1}=0.65<1$ and $\max \boldsymbol{a}=$ $\|\boldsymbol{a}\|_{\infty}=0.2<1 / 4$

and initial conditions $\boldsymbol{Y}=\left[\begin{array}{llll}1 & 5 & -6 & 3\end{array}\right]$.

Note that case i) corresponds to the strict FPI convergence, whereas in the case ii) we can expect pseudoperiodic behavior. The diagram of the values of (7) are shown in Fig. 1. The solid line in Fig. 1, which represents the case i) i.e. $\|a\|_{\infty}=0.7>1 / 4$, decays monotonically 
toward zero for lags $n>4$, i.e. after the initial values are processed by (7). The dashed line in Fig. 1, which represents the case ii) i.e. $\|\boldsymbol{a}\|_{\infty}=$ $0.2<1 / 4$, decays in an oscillatory way toward zero for lags $n>4$, which confirms Theorem 2.

\section{A. Stability result for $\sum_{i=1}^{m} a_{i}=1$}

Let us finally consider the case with constant parameter vector $a=$ $\left[a_{1}, \cdots, a_{m}\right]^{T}$, where $\|\boldsymbol{a}\|_{1}=1$. In that case, the matrix $\boldsymbol{A}$ from (3) becomes a stochastic matrix [12], [13], since each of its rows is a probability vector, i.e. each entry of a row is nonnegative and the sum of the entries in each row is unity. In addition, sets of real stochastic matrices are compact convex sets [6]. This being the case, the process (3) can be rewritten as

$$
\boldsymbol{Y}(n+1)=\boldsymbol{A} \boldsymbol{Y}(n)=\boldsymbol{A}^{2} \boldsymbol{Y}(n-1) \cdots=\boldsymbol{A}^{n} \boldsymbol{Y}(0)
$$

which means that the dynamics of (3) are fully described by its initial state $\boldsymbol{Y}(0)$, and the system matrix $\boldsymbol{A}$. In addition, since the product of two stochastic matrices is a stochastic matrix, and the stochastic matrix $\boldsymbol{A}$ is a regular stochastic matrix, it has a unique fixed vector $\boldsymbol{t}=\left[t_{1}, \cdots, t_{m}\right]$ such that $[12]$

$$
\boldsymbol{t} A=\boldsymbol{t}
$$

which is a probability vector itself, i.e. $\|\boldsymbol{t}\|_{1}=1$. In that case

$$
\boldsymbol{A}^{n}=\left[\begin{array}{cccc}
a_{1} & a_{2} & \cdots & a_{m} \\
1 & 0 & \cdots & 0 \\
\vdots & \vdots & \ddots & \vdots \\
0 & \cdots & 1 & 0
\end{array}\right]^{n} \stackrel{n \rightarrow \infty}{\rightarrow}\left[\begin{array}{cccc}
t_{1} & t_{2} & \cdots & t_{m} \\
t_{1} & t_{2} & \cdots & t_{m} \\
\vdots & \vdots & \ddots & \vdots \\
t_{1} & t_{2} & \cdots & t_{m}
\end{array}\right]
$$

Lemma 2: The process (7) with the constant coefficient vector $\boldsymbol{a}=$ $\left[a_{1}, \cdots, a_{m}\right]^{T}$, where $a_{i} \geq 0, i=1, \cdots m$ converges to

i) $\left|y_{\infty}\right|=\left|\Sigma_{i=1}^{m} t_{i} y(n-i)\right| \geq 0$ for $\|\boldsymbol{a}\|_{1}=1$

ii) $y_{\infty}=0$ for $\|a\|_{1}<1$

from any finite initial state $\boldsymbol{Y}(0)$.

Proof:

i) Since from (11), the matrix $\boldsymbol{A}^{n}$ approaches a constant matrix with positive entries, and with rows being fixed vectors of the matrix $\boldsymbol{A}$, the quantity $y_{\infty}=\left.y(n)\right|_{n \rightarrow \infty}$ becomes a linear combination of its previous values, with strictly positive coefficients. That means that, in a general case, $y_{\infty}>0$, with $y_{\infty}=0$ if and only if the vectors $\boldsymbol{t}$ and $\boldsymbol{a}$ are orthogonal, i.e. $\boldsymbol{t}^{T} \boldsymbol{a}=0$.

ii) Since the rows $(2-m)$ in $\boldsymbol{A}$ represent the shift operator, the dynamics of the system (3) rest upon the first row of $\boldsymbol{A}$, which is $\boldsymbol{a}^{T}$. The condition $\|\boldsymbol{a}\|_{1}<1$ introduces decay into the system, and the output of the system converges to zero.

Example 2: Check Lemma 2 for

i) $\boldsymbol{a}=\left[\begin{array}{llll}0.15 & 0.2 & 0.5 & 0.15\end{array}\right]$ where $\|\boldsymbol{a}\|_{1}=1$

ii) $\boldsymbol{a}=\left[\begin{array}{llll}0.15 & 0.1 & 0.5 & 0.15\end{array}\right]$ where $\|\boldsymbol{a}\|_{1}=0.9<1$

and the initial condition $\boldsymbol{Y}=\left[\begin{array}{llll}1 & 5 & -6 & 3\end{array}\right]$.

Let us set up the recursion (3), where the first row in $\boldsymbol{A}$ should be $\boldsymbol{a}$ from i) or ii), and plot the resulting values $y(n), n=1,2, \cdots$, as shown in Fig. 2. The diagram in Fig. 2 confirms the claim from Lemma 2, since for i) where $\|\boldsymbol{a}\|_{1}=1$, the iteration (7) converges to a nonzero value, whereas for the case ii) where $\|a\|_{1}<1$, the iteration (7) converges to the value of zero.

\section{B. Examples}

We present some results for the convergence in the geometric and norm sense of the approach presented so far.

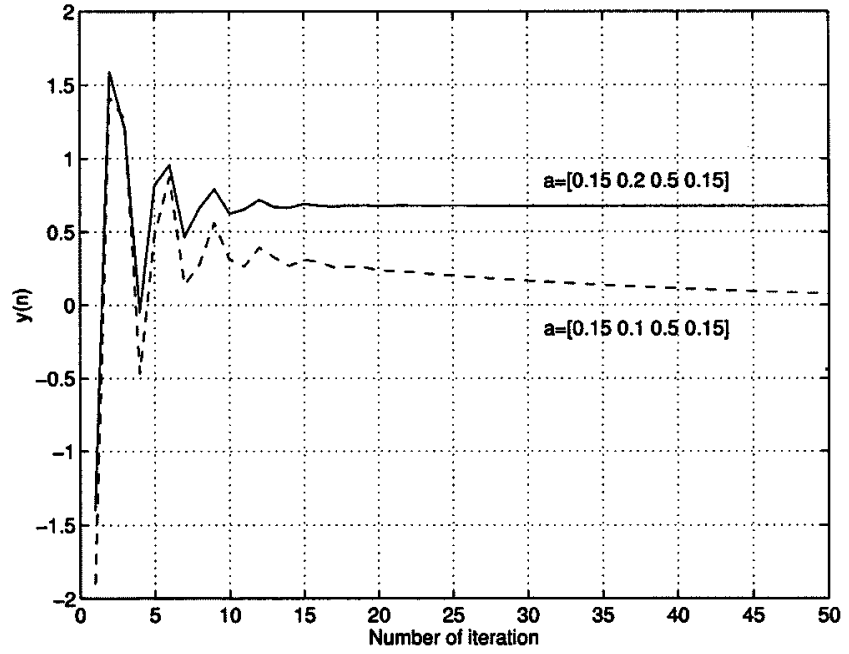

Fig. 2. Convergence of relaxation for $\|\boldsymbol{a}\|_{1}=1$ and $\|\boldsymbol{a}\|_{1}<1$.
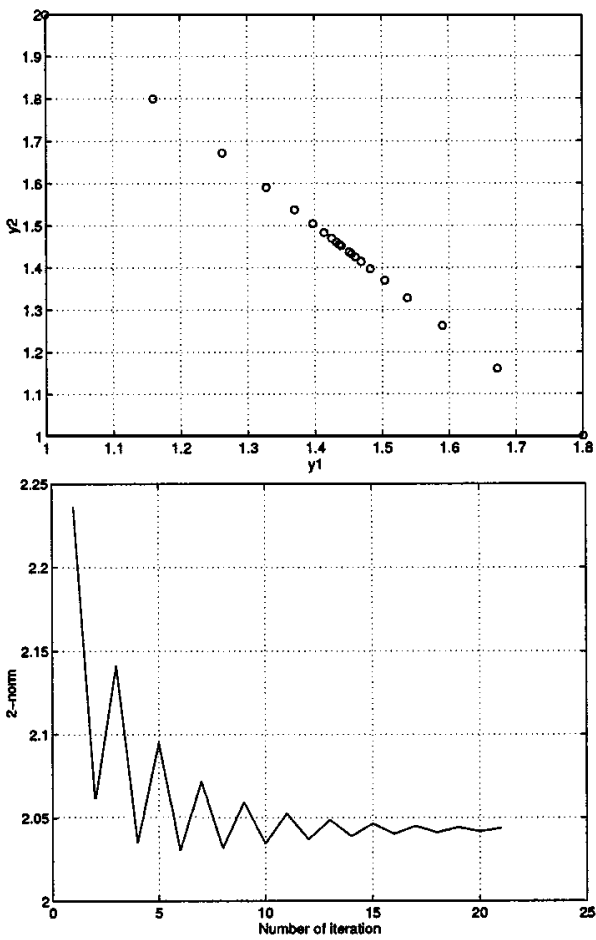

Fig. 3. Convergence of the process $\boldsymbol{Y}(n)=A Y(n-1)$.

For the matrix $a_{11}=0.2, a_{12}=0.8, a_{21}=1, a_{22}=0$, and the initial conditions $Y=\left[\begin{array}{ll}1 & 2\end{array}\right]$, the geometric convergence of the vector $Y$ in the plane, and the convergence of its $\|\cdot\|_{2}$ norm are shown in Fig. 3. As $\|\boldsymbol{a}\|_{1}=1$, and the entries in $\boldsymbol{a}$ are nonnegative, the points $\boldsymbol{Y}(n)=\left[y_{1}(n), y_{2}(n)\right]$ converge toward a point, forming a line in the plane (convexity). The convergence in the norm is oscillatory, and toward a nonzero point (Lemma 2).

For the matrix $a_{11}=-0.2, a_{12}=0.8, a_{21}=1, a_{22}=0$, and the initial conditions $Y=\left[\begin{array}{ll}1 & 2\end{array}\right]$, the geometric convergence of the vector $Y$ in the plane, and the convergence of its $\|\cdot\|_{2}$ norm are shown in Fig. 4. Here, $\|\boldsymbol{a}\|_{1}=1$, but not all elements of $\boldsymbol{a}$ are nonnegative. The process (1) converges in the norm, but not in the geometric sense, where it achieves its limit cycle, for there are two distinct points, with the same norm, to which the process converges. That is because, with both the positive and negative entries in $a$, the convexity is violated, and the functions and adjacent sets are affine, rather than convex. 

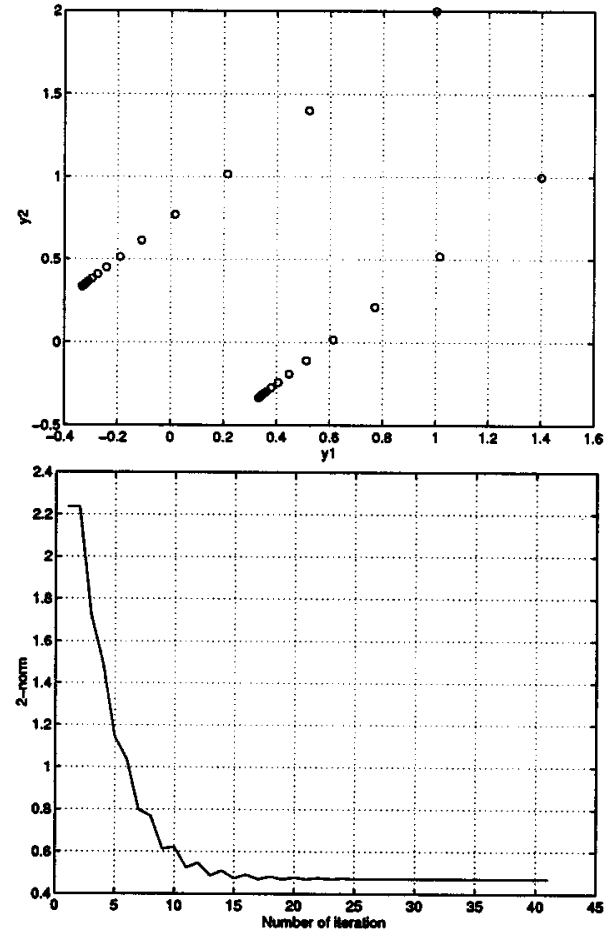

Fig. 4. Convergence of the process $\boldsymbol{Y}(n)=\boldsymbol{A} \boldsymbol{Y}(n-1)$.

Claim 1: For the system (1), with $\|\boldsymbol{a}\|_{1}=1$, the convergence in the norm does not imply the convergence in the geometric sense.

\section{CONCLUSION}

Based upon the results in [1], for the case $\|a\|_{1}<1$, we have shown that all the statements given in [1] can be derived simply as a consequence of convexity and affinity of the sets of initial values of the signal considered, and the filter parameters, if all the entries in $\boldsymbol{a}$ are nonnegative. The convexity property, together with the contraction mapping imposed on the filter equation, allows derivation of the conditions of the pseudoperiodic and aperiodic GAS, as well as uniform GAS. The values to which the processes converge in the cases of aperiodic and pseudoperiodic convergence have been found. That has been achieved through the $\|\cdot\|_{1}$ and $\|\cdot\|_{\infty}$ norm of the coefficient vector $\boldsymbol{a}$. In this approach, the values of the coefficient vector are allowed to change freely within the convex set $\mathcal{A}$ of all the allowable values of $\boldsymbol{a}$. In addition, we have derived corresponding results for the case $\|a\|_{1} \leq 1$, using the state space approach, and the fixed-point theory, and have shown that the convergence in the norm, does not necessarily imply geometric convergence. It has been shown that the case when $\boldsymbol{a}$ has only one positive entry, and $\|a\|_{1}=1$ leads to occurrence of limit cycles. The examples presented fully support our approach.

\section{REFERENCES}

[1] P. Bauer, M. Mansour, and J. Duran, "Stability of polynomials with time-varying coefficients," IEEE Transactions on Circuits and Systems-I: Fundamental Theory and Applications, vol. 40, no. 6, pp. 423-426, 1993.

[2] T. Kailath, Linear Systems: Prentice-Hall, 1980.

[3] S. Haykin, Adaptive Filter Theory, 3rd ed: Prentice-Hall, 1996.

[4] J. P. LaSalle, The Stability and Control of Discrete Processes. Berlin, Germany: Springer-Verlag, 1986
[5] J. H. Wilkinson, The Algebraic Eigenvalue Problem: Oxford University Press, 1965

[6] R. A. Horn and C. A. Johnson, Matrix Analysis: Cambridge University Press, 1985.

[7] J. E. Dennis Jr. and R. B. Schnabel, Numerical Methods for Unconstrained Optimization an Nonlinear Equations: Prentice-Hall Series in Computational Mathematics, 1983.

[8] E. Zeidler, Nonlinear Functional Analysis and Its Applications: Springer-Verlag, 1986, vol. 1.

[9] S. Lipschutz, General Topology: McGraw-Hill International, 1981.

[10] G. E. Box and G. M. Jenkins, Time Series Analysis: Forecasting and Control, 2nd ed: Holden-Day, 1976.

[11] J. Makhoul, "Linear prediction: A tutorial overview," in Proceedings of the IEEE, vol. 63, 1975, pp. 561-580.

[12] S. Lipschutz, Theory and Problems of Probability: McGraw-Hill, 1965.

[13] H. Stark and J. W. Woods, Probability, Random Processes and Estimation Theory for Engineers. Englewood Cliffs, NJ: PrenticeHall, 1986.

\section{Adaptive Control of Chaotic Dynamical Systems Using Invariant Manifold Approach}

\author{
Yu-Ping Tian and Xinghuo Yu
}

\begin{abstract}
In this brief, an adaptive chaos control method is developed for stabilizing chaotic systems at their unknown equilibrium(s) using the invariant manifold theory. The developed method overcomes the problem that the equilibrium(s) of the chaotic systems are dependent on the unknown system parameters, which makes direct application of the conventional adaptive control difficult. Further development of the adaptive chaos control is undertaken for the situation where the parameter estimates are only allowed to vary within a bounded set due to the sensitivity of chaotic systems to parameter variations. A sufficient condition for convergence of system states and parameter estimates is obtained. The design method developed then is applied to stabilizing the Lorenz chaotic system at an unknown equilibrium. Both mathematical and computational results have demonstrated the effectiveness of this method.
\end{abstract}

Index Terms-Adaptive control, chaos control, invariant manifolds, Lorenz system, Lyapunov method.

\section{INTRODUCTION}

Controlling chaos has attracted more and more attention recently, and has become a very active multidisciplinary research area involving physics, biology, mathematics, and engineering. Various control strategies for chaos control have been developed, e.g., [1]-[5] and references therein.

The basic assumption of the existing chaos control results is that the system parameters are known a priori. Unlike most conventional control systems whose equilibriums are assumed known and fixed regardless of values of the system parameters, the equilibriums of chaotic systems are a function of their system constant parameters. This suggests that, when the constant parameters are not precisely known (or

Manuscript received August 25, 1998; revised June 14, 2000. This work was supported in part by the National Key Project and the National Natural Science Foundation of China, and by the Australian Research Council.

Y.-P. Tian is with the Department of Automatic Control, Southeast University, Nanjing 210096, China.

$\mathrm{X}$. Yu is with the Faculty of Informatics and Communication, Central Queensland University, Rockhampton QLD 4702, Australia.

Publisher Item Identifier S 1057-7122(00)08338-0. 